\title{
Ta Doping Effect on Structural and Optical Properties of InTe Thin Films
}

\author{
Chunmin Liu ${ }^{1}$, Yafei Yuan ${ }^{1,2}$, Xintong Zhang ${ }^{1}$, Jing Su ${ }^{1}$, Xiaoxiao Song ${ }^{1}$, Hang Ling ${ }^{1}$, \\ Yuanjie Liao ${ }^{1}$, Hao Zhang ${ }^{1} \mathbb{D}$, Yuxiang Zheng ${ }^{1}$ and Jing $\mathrm{Li}^{1}{ }^{1} * \mathbb{E}$ \\ 1 Department of Optical Science and Engineering, Shanghai Ultra-Precision Optical Manufacturing \\ Engineering Center, Fudan University, Shanghai 200433, China; 18110720032@fudan.edu.cn (C.L.); \\ 15110720031@fudan.edu.cn (Y.Y.); 17110720006@fudan.edu.cn (X.Z.); 16110720001@fudan.edu.cn (J.S.); \\ 19110720013@fudan.edu.cn (X.S.); 18210720094@fudan.edu.cn (H.L.); 19210720009@fudan.edu.cn (Y.L.); \\ zhangh@fudan.edu.cn (H.Z.); yxzheng@fudan.edu.cn (Y.Z.) \\ 2 Department of Electronic Engineering, Center for Intelligent Medical Electronics, Fudan University, \\ Shanghai 200433, China \\ * Correspondence: lijing@fudan.edu.cn
}

Received: 19 August 2020; Accepted: 17 September 2020; Published: 21 September 2020

\begin{abstract}
The objective of this work was to study the influence of Ta doping on the structural, transmittance properties, linear absorption parameter, and nonlinear absorption properties of InTe thin films. The as-deposited samples with different Ta doping concentrations were prepared by a magnetron co-sputtering technique and then annealed in nitrogen atmosphere. Structural investigations by X-ray diffraction revealed the tetragonal structure of InTe samples and that the crystallinity decreases with increasing Ta doping concentration. Further structural analysis by Raman spectra also showed good agreement with $\mathrm{X}$-ray diffraction results. The Ta doping concentration and sample thickness determined by energy-dispersive $X$-ray spectroscopy and scanning electron microscopy increased as Ta dopant increased. In addition, X-ray photoelectron spectroscopic was carried out to analyze the chemical states of the elements. UV-VIS-NIR transmittance spectra were applied to study the transmittance properties and calculate the linear absorption coefficient. Due to Burstein-Moss effect, the absorption edge moved to shorter wavelengths. Meanwhile, the values of band gap were found to increase from $1.71 \pm 0.02 \mathrm{eV}$ to $1.85 \pm 0.01 \mathrm{eV}$ with the increase of Ta doping concentration. By performing an open aperture Z-scan technique, we found that all Ta-doped InTe samples exhibited two-photon absorption behaviors. The nonlinear optical absorption parameters, such as modulation depth, two-photon absorption coefficient, and two-photon absorption cross-section, decrease with increasing Ta concentration, whereas the damage threshold increases from $176 \pm 0.5 \mathrm{GW} / \mathrm{cm}^{2}$ to $242 \pm$ $0.5 \mathrm{GW} / \mathrm{cm}^{2}$. These novel properties show the potential for applications in traditional optoelectronic devices and optical limiters.
\end{abstract}

Keywords: Ta-doped InTe thin film; mixed-valence compound; linear optical absorption; Z-scan; two-photon absorption

\section{Introduction}

Chalcogenide thin films have drawn considerable interest in recent years, owing to their novel properties and broad range of applications in high-performance optical and electric fields, such as phase change random-access memory (PRAM), resistance random-access memory (ReRAM), nonlinear optical absorption (NOA) devices, thin-film solar cells, and so on [1-4]. Among them, group IIIA metal chalcogenide (GIIIAMC) is an important member. On the basis of stoichiometric composition, GIIIAMC can be simply divided into two categories: One is AB type, such as InSe, InTe, GaSe, and GaTe, and the 
other is $\mathrm{A}_{2} \mathrm{~B}_{3}$ type, for example, $\mathrm{In}_{2} \mathrm{Se}_{3}, \mathrm{In}_{4} \mathrm{Se}_{3}$, and $\mathrm{In}_{2} \mathrm{Te}_{3}$ [5]. As mentioned, indium telluride (InTe), better written as $\mathrm{In}^{1+} \mathrm{In}^{3+} \mathrm{Te}_{2}{ }^{2-}$, is a mixed-valence compound belonging to the $\mathrm{AB}$ type with a chain TlSe-type structure (space group I4/mcm) [6]. The ionic and covalent substructures coexist in this structure, that is, the trivalent $\mathrm{In}^{3+}$ ions are tetrahedrally coordinated by four $\mathrm{Te}^{2-}$ ions, forming covalent In-Te bonds. On the other hand, the monovalent $\operatorname{In}^{1+}$ ions are surrounded by eight $\mathrm{Te}^{2-}$ ions in a tetragonal antiprismatic arrangement [7]. The two different crystallographic positions of distinct $\mathrm{In}^{3+}$ and $\operatorname{In}^{1+}$ prevent the free transfer of electrons from $\operatorname{~In}^{1+}$ to $\operatorname{In}^{3+}[8]$.

For applications, Jana et al. reported that the room temperature lattice thermal conductivity of InTe is as low as $0.76 \mathrm{~W} /(\mathrm{m} \cdot \mathrm{K})$, which is much smaller than those of other Te-based compounds, such as $2.9 \mathrm{~W} /(\mathrm{m} \cdot \mathrm{K})$ of GeTe [9]. This characteristic is beneficial to thermoelectric material development since it can be applied to the thermoelectric solid state cooling technologies and waste heat power generation [10]. For optical absorption performance, Peng et al. calculated that the upper limit of the energy conversion efficiency of sunlight of the InTe reached as large as $6.4 \%$, which was superior to those of the transition metal dichalcogenides (TMDs) with a predicted energy conversion efficiency of less than $0.4 \%[11,12]$. In the field of PRAM, the contaminants induced errors and low phase-change temperature $\left(<453 \mathrm{~K}\right.$ ) limited the usage of some compounds such as $\mathrm{Ge}-\mathrm{Sb}-\mathrm{Te}$ and $\mathrm{Ge}_{2} \mathrm{Sb}_{2} \mathrm{Te}_{5}$ [13]. Sugiyama et al. reported the phase-change temperature of InTe thin films over $523 \mathrm{~K}$, which overcomes such a disadvantage [14]. Especially, Yuan et al. found that InTe, a promising optical limiting material, has superior third-order nonlinear characteristics [15]. However, the change of InTe damage threshold is not clear. Although InTe has been widely used in both switching devices and solar cells, there is still some opportunities for better performance, such as forming heterojunctions with other materials, adjusting the proportion of elements, and doping foreign elements [16-18]. Among them, doping is a common method. For instance, several Ti-doped chalcogenides have been well studied in theory and experiments, but little has been reported about other inert metal elements $[15,19]$. In the present work, tantalum, an inert metal element, is chosen as the dopant because it has a higher solid solubility and can induce high electrical conductivity while preserving high transmittance [20,21]. For example, Ta has been used as an outstanding candidate for improving stability and thermal properties of chalcogenide thin films [22]. In addition, though many studies relating to the growth, structural characteristics, pressure dependent behaviors, and thermoelectric properties of InTe films have been reported $[6,7,9,18]$, there are few studies on the NOA properties of intrinsic or doped InTe.

Therefore, combining these two considerations, the Ta element is chosen as the dopant to extensively study the effects on the structural, transmittance, linear optical absorption, and NOA properties of InTe thin films. To gain insights into the adjustability of InTe films with the Ta dopant, various properties of Ta-doped InTe films, such as the crystal structure, optical band gap, nonlinear absorption parameters, etc., are discussed in-depth, as follows. The results are important for using the InTe material in many applications.

\section{Experimental Details}

A LAB600sp-type magnetron co-sputtering system (Leybold Optics GmbH, Dresden, Germany), was adopted to prepare film samples. Through magnetron co-sputtering, the Ta-doped InTe thin films was deposited on fused quartz and $\mathrm{Si}(100)$ substrates with the same conditions. The sputtering power of the InTe target was set to constant value of $200 \mathrm{~W}$, and that of the Ta target was changed from 0 to $25 \mathrm{~W}$. The background and working pressures in the chamber were controlled at $7 \times 10^{-6} \mathrm{mbar}$ and $2.8 \times 10^{-3}$ mbar, respectively. The purity of both targets is $99.99 \%$. All the substrates were kept at room temperature during the whole sputtering process. After deposition, a set of samples was annealed at $450{ }^{\circ} \mathrm{C}$, for $30 \mathrm{~min}$, in a flowing nitrogen atmosphere.

The crystallization characteristics of the samples were determined by X-ray diffraction (XRD) with the $\mathrm{Cu}-\mathrm{K} \alpha(1.54056 \AA$ ) radiation (Bruker D8 ADVANCE) (Karlsruhe, Germany), in the $2 \theta$ angles range of $10-60^{\circ}$, with a step of $0.02^{\circ}$. The scanning electron microscopy (SEM) (Hillsboro, Oregon, USA) mapping scan and energy dispersive X-ray spectroscopy (EDS) (EDAX Inc., New Jersey, USA) 
elemental analysis were performed via an FEI Siron 200 ultra-high resolution scanning electron microscope. The maximum magnification is $\times 10^{6}$, and the resolution is $1 \mathrm{~nm} @ 1 \mathrm{kV}$. Raman spectra of the samples were tested by using the Nanofinder 30(Tokyo Instruments, Tokyo, Japan). A double-beam UV-VIS-NIR spectrophotometer (Shimadzu UV-3600) (Shimadzu Co. Ltd., Kyoto, Japan), country) was employed to determine the transmission spectroscopic characteristics. Chemical components and bonding analyses were carried out by using X-ray photoelectron spectroscopy (XPS; Kratos Axis Ultra Dld) (Shimadzu Co. Ltd., Kyoto, Japan).

In order to measure the NOA properties of Ta-doped InTe thin films, a single beam open-aperture (OA)-mode Z-scan method was built for it. The details of the setup were discussed in our previous work [3]. All the samples were excited by a pulsed laser at $800 \mathrm{~nm}$ excitation wavelength with $100 \mathrm{fs}$ pulse duration and $1 \mathrm{kHz}$ repetition rate from the Ti-sapphire regenerative amplifier system (Spectra Physics, Spitfire Ace) (California, USA). In this system, the Rayleigh length $\left(Z_{0}=\frac{\pi \omega^{2}}{\lambda}\right)$ and the beam radius, $\omega$, at the focal point were $4 \mathrm{~mm}$ and $32 \mu \mathrm{m}$, respectively. Each sample thickness was much smaller than the diffraction length of focused beam. Therefore, the samples could be regarded as "thin" films [23]. In the process of measurement, each thin-film sample was moved along the $\mathrm{Z}$ axis (propagation direction), to be driven by a stepping motor. Through calibration tests, our system met the requirements of Z-scan theory [3].

\section{Results and Discussion}

\subsection{XRD Analysis}

Four annealed Ta-doped InTe thin film samples are named S1-S4, with increasing deposition powers of Ta target, respectively. Figure 1a shows the XRD patterns of the S1-S4 samples. The sharp diffraction peaks suggest high crystallinity and the polycrystalline nature of the films. All the samples show the characteristic peaks of tetragonal phase of InTe (JCPDS No: 30-0636) with lattice parameters a $=\mathrm{b}=8.454 \AA$ and $\mathrm{c}=7.152 \AA$, indicating the formation of better crystalline InTe films. This structure is consistent with some researches [6,7]. The absence of Ta diffraction peaks indicates that the Ta dopant is well mixed in the lattice of InTe; similar results have been reported by previous work [24].
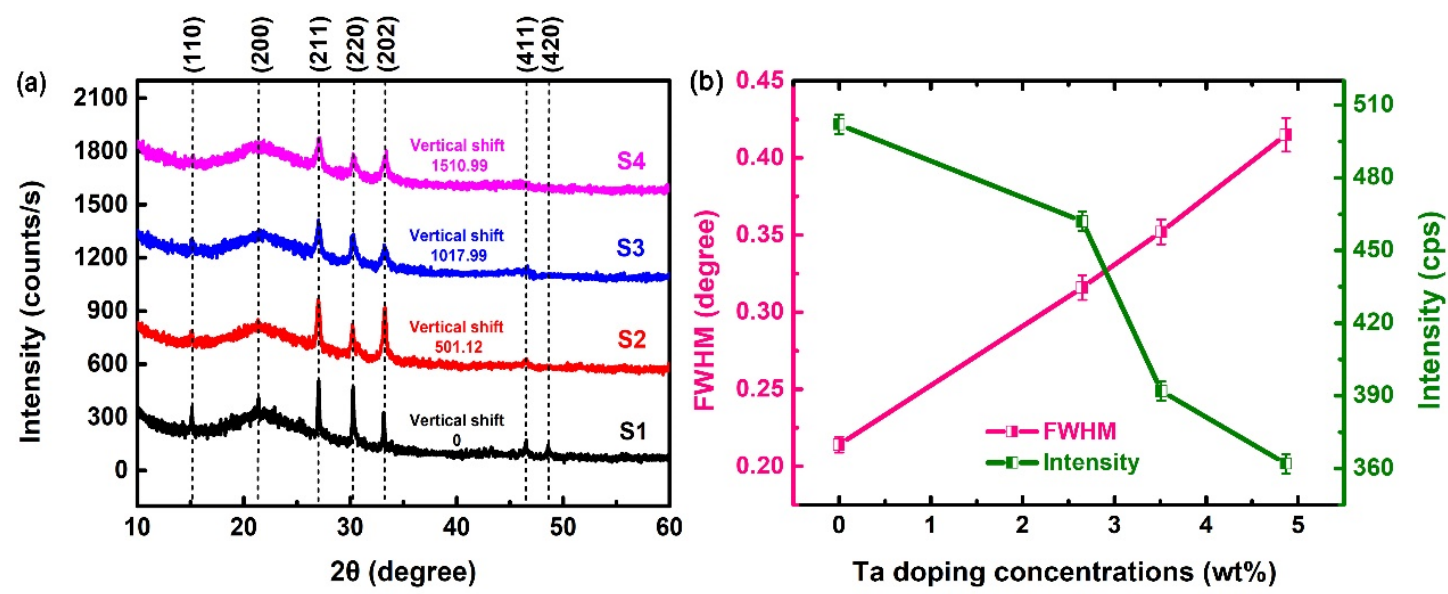

Figure 1. (a) XRD patterns of Ta-doped InTe thin films S1-S4; (b) variations of FWHM and intensity of the (211) peak.

As presented in Figure 1b, S1-S4 exhibit a preferred (211) orientation, and the intensity of this diffraction peak decreases with the increase of the Ta dopant concentration, while the full width at half maximum (FWHM) of the (211) peak increases accordingly. In addition, Table 1 gives the 
variations of the FWHM and crystallite sizes. The mean crystallite size $(D)$ is estimated from the Scherrer Equation [25]:

$$
D=\frac{0.9 \lambda}{B \cos \theta}
$$

where $\lambda$ is the wavelength of $X$-ray radiation $(1.54056 \AA), B$ represents the full width at half maximum of the peak, and $\theta$ refers to the angle of Bragg diffraction. The calculated values of crystallite sizes decrease with increasing Ta dopant concentration. It indicates that the increase of Ta dopant concentration will reduce the crystallinity of the film samples. It should be attributed to stress, as well as the formation of defects or disorders due to segregation of dopants on the grain boundaries [24].

Table 1. Preparation parameters and the variations of the full width at half maximum (FWHM) and crystallite sizes.

\begin{tabular}{ccccc}
\hline Sample & InTe Power $(W)$ & Ta Power $(W)$ & FWHM (degree) & Crystallite Size (nm) \\
\hline S1 & 200 & 0 & $0.2142 \pm 0.0052$ & $38.2 \pm 0.8$ \\
S2 & 200 & 15 & $0.3167 \pm 0.0078$ & $25.8 \pm 0.6$ \\
S3 & 200 & 20 & $0.3520 \pm 0.0079$ & $23.2 \pm 0.5$ \\
S4 & 200 & 25 & $0.4144 \pm 0.0110$ & $19.7 \pm 0.5$ \\
\hline
\end{tabular}

\subsection{Raman Spectral Analysis}

As an additional characterization, the Ta-doped InTe thin films were studied by Raman spectroscopy, and the results are shown in Figure 2. There are five vibrational bands located at $\sim 102, \sim 125, \sim 142, \sim 194$, and $\sim 267 \mathrm{~cm}^{-1}$. Notable peaks for these samples and phonon modes are listed in Table 2. Our Raman spectrum of the intrinsic InTe film matches well with that in other works [26,27]. As shown in Figure 2, the two weak peaks $\sim 194$ and $\sim 267 \mathrm{~cm}^{-1}$ gradually disappear as the concentration of the dopant increases. This observation could be attributed to the destruction of the long Te chain by the doping of Ta. Similar experimental phenomena have been reported in the literature [15]. The peak fitting results of the three main peaks are shown in Figure 3. The phonon modes at about 125 and $142 \mathrm{~cm}^{-1}$ are assigned to $\mathrm{A}_{1 \mathrm{~g}}$ and $\mathrm{E}_{\mathrm{g}}$ symmetry, respectively. The phonon assignment matches well with the previously reported Raman studies of InTe by Nizametdinova [28]. The $\mathrm{A}_{1 \mathrm{~g}}$ mode is due to the motion of Te atoms alone, while for $\mathrm{E}_{\mathrm{g}}$ mode, Te and $\mathrm{In}^{3+}$ atoms vibrate in opposite directions. Based on the work of Rajaji et al. [7], in these two modes, $\operatorname{the}^{1{ }^{1+}}$ atoms do not exhibit any movement. Besides, the phonon mode at about $102 \mathrm{~cm}^{-1}$ is assigned to lower $\mathrm{E}_{\mathrm{g}}$ mode, which agrees with the Raman spectra recorded by Pine et al. [26]. Comparing S1-S4, the phonon mode at about $142 \mathrm{~cm}^{-1}$ moves to about $138 \mathrm{~cm}^{-1}$ after Ta doping, and the intensity decreases with the increase of Ta dopant concentration. This should be due to the destruction of translational symmetry as a result of generation of defects or substitution.

\subsection{SEM, EDS, and XPS Analysis}

The film thickness estimated from SEM images of S1-S4 are $306 \pm 1,327 \pm 1,338 \pm 1$, and $356 \pm 1 \mathrm{~nm}$, respectively. The SEM images of S2 and S3 are shown in Figure 4. In the SEM images, the ambient, main Ta-doped InTe layer, and Si substrate are clearly distinguished. In addition, it should be noted that the protrusions in the SEM image for S3 are the marginal remnants left behind when cutting the sample. The composition of the Ta-doped InTe thin films from EDS are listed in Table 3. Obviously, both the thickness and the weight percentage of Ta dopant increase as the sputtering power of the Ta target increases. 


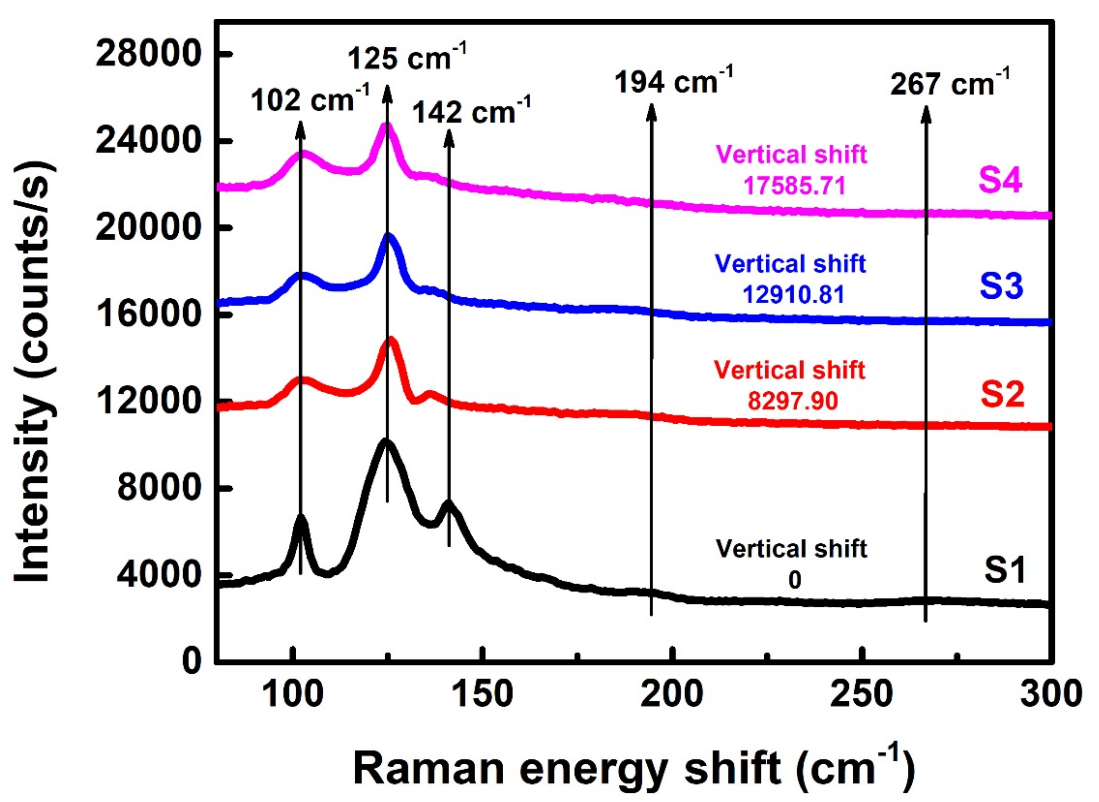

Figure 2. Raman spectra of Ta-doped InTe thin films S1-S4.

Table 2. Raman peaks of Ta-doped InTe thin films and related phonon modes.

\begin{tabular}{ccccccccccc}
\hline Reference & \multicolumn{10}{c}{ Characteristic Wavenumbers $\mathbf{( c m}^{-\mathbf{1}}$ ) } \\
\hline This Work & - & - & $\mathbf{1 0 2} \pm \mathbf{1}$ & $\mathbf{1 2 5} \pm \mathbf{1}$ & $\mathbf{1 4 2} \pm \mathbf{1}$ & - & - & $\mathbf{1 9 4} \pm \mathbf{1}$ & - & $\mathbf{2 6 7 \pm \mathbf { 1 }}$ \\
\hline $\mathrm{Te}[26]$ & - & - & 105 & 123 & 143 & - & - & - & - & - \\
$\mathrm{Te}[27]$ & - & - & - & 120 & 140 & - & 176 & - & - & 260 \\
$\mathrm{InTe}[28]$ & 46 & 86 & - & 126 & 139 & - & - & - & - & - \\
$\mathrm{In}_{2} \mathrm{Te}_{3}[29]$ & - & - & 103 & 125 & 142 & 157 & - & 194 & 225 & - \\
$\mathrm{CuInTe}_{2}[30]$ & - & - & - & 123 & 143 & - & 171 & - & 220 & 267 \\
\hline
\end{tabular}
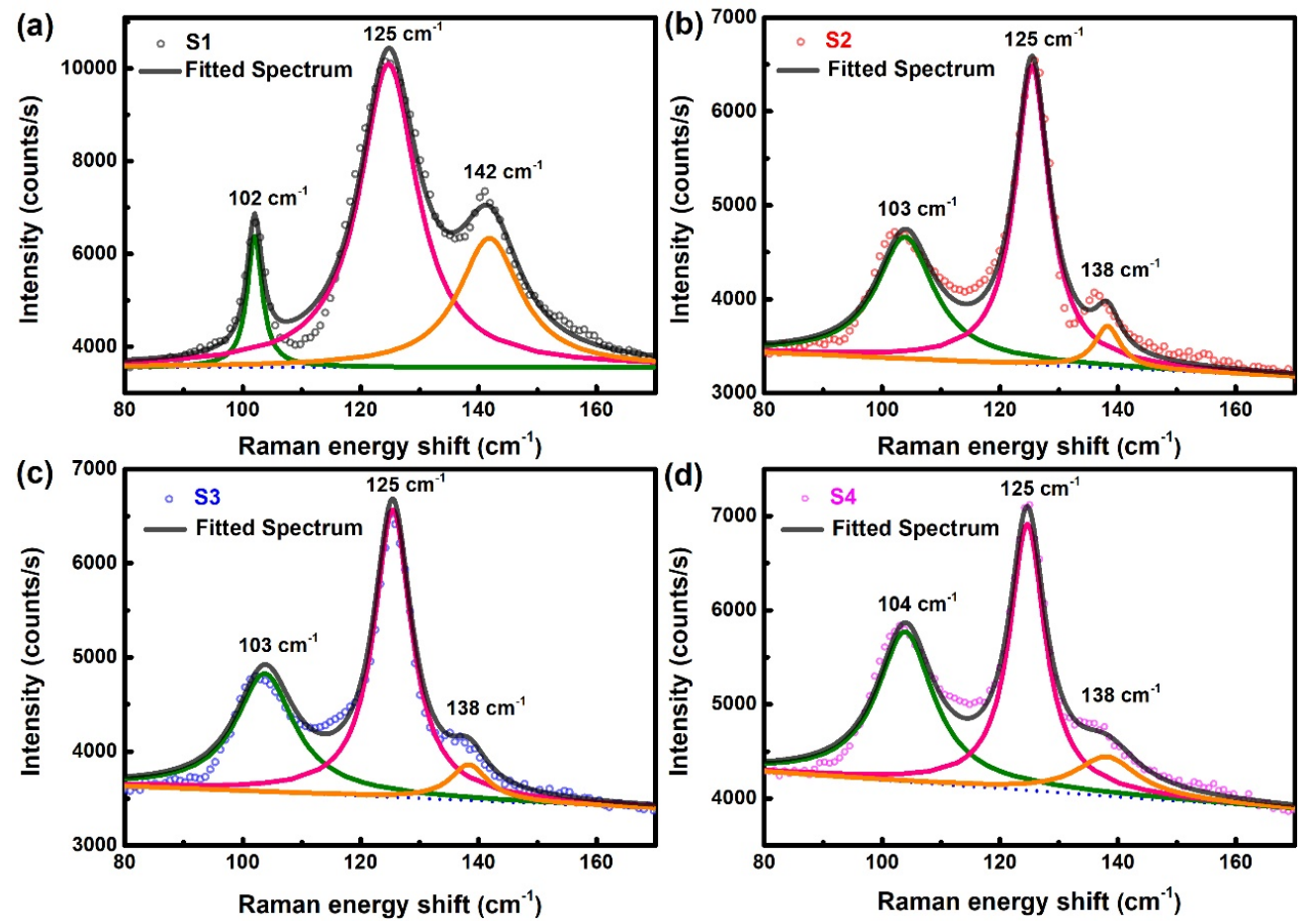

Figure 3. The peak fitting Raman spectra of Ta-doped InTe thin films: (a) S1, (b) S2, (c) S3, (d) S4. 


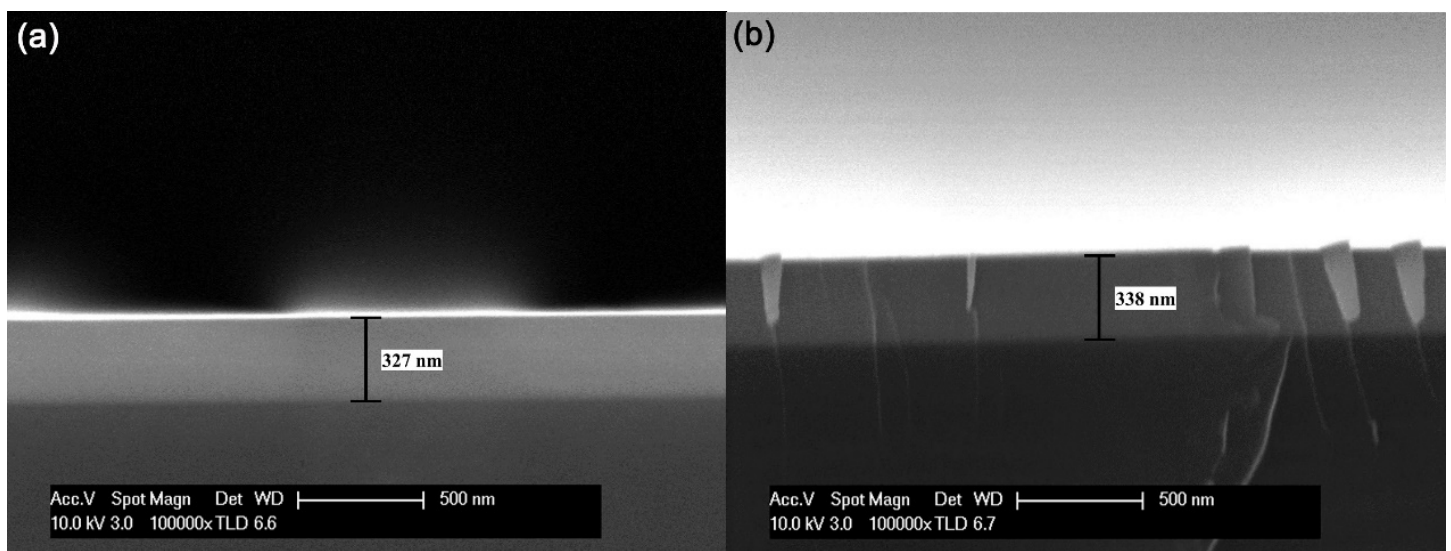

Figure 4. The cross-sectional SEM images of (a) S2 and (b) S3.

Table 3. The composition and thickness results of Ta-doped InTe thin films.

\begin{tabular}{cccccc}
\hline Sample & Ta Power (W) & In (wt. $\%)$ & Te (wt.\%) & Ta (wt.\%) & Thickness (nm) \\
\hline S1 & 0 & 51.07 & 48.93 & 0 & $306 \pm 1$ \\
S2 & 15 & 50.19 & 47.16 & 2.65 & $327 \pm 1$ \\
S3 & 20 & 48.12 & 48.37 & 3.51 & $338 \pm 1$ \\
S4 & 25 & 49.31 & 45.81 & 4.87 & $356 \pm 1$ \\
\hline
\end{tabular}

The X-ray photoelectron spectroscopic measurement was performed to analyze the chemical states of the samples. The 3D high-resolution spectrum of S3 in Figure 5a shows two strong peaks at $\sim 444.4$ and $\sim 452.0 \mathrm{eV}$, which can be assigned to In $3 \mathrm{~d}_{5 / 2}$ and $\mathrm{In} 3 \mathrm{~d}_{3 / 2}$ with a spin-orbit splitting of $7.6 \mathrm{eV}$. Similarly, as seen in Figure $5 b$, two strong peaks corresponding to Te $3 \mathrm{~d}_{5 / 2}$ and Te $3 \mathrm{~d}_{3 / 2}$ states are observed at $\sim 572.5$ and $\sim 582.9 \mathrm{eV}$, respectively. The XPS results of In and Te are consistent with the InTe crystal [31]. Four prominent Ta $4 \mathrm{f}$ states are observed in Figure 5c. The former peaks of Ta $4 \mathrm{f}_{7 / 2}$ at $\sim 26.3 \mathrm{eV}$ and $\mathrm{Ta} 4 \mathrm{f}_{5 / 2}$ at $\sim 28.3 \mathrm{eV}$ are attributed to $\mathrm{Ta}^{5+}$ state, which should be introduced by the resonance bond. Resonance happens when more than one valence-bond structure can be written for a molecule or ion. Then, the true structure is a blend of all the different possible structures [32]. In this work, the combination of Ta and Te elements forms a complex structure; thus, $\mathrm{Ta}^{5+}$ state appears.

The latter peaks at $\sim 22.5 \mathrm{eV}\left(\mathrm{Ta}_{4} \mathrm{f}_{7 / 2}\right)$ and $\sim 24.4 \mathrm{eV}\left(\mathrm{Ta}_{4} \mathrm{f}_{5 / 2}\right)$ correspond to a $\mathrm{Ta}^{4+}$ state, indicating the formation of $\mathrm{TaTe}_{2}$. The same experimental results were reported in a previous study [33]. These results suggest that the Ta element forms both $\mathrm{Ta}^{5+}$ and $\mathrm{Ta}^{4+}$ chemical states when Ta is doped into the InTe film.

\subsection{Linear Optical Analysis}

The optical transmittance spectra of the Ta-doped InTe thin films in the spectrum range of 300-3000 nm are displayed in Figure 6a. The transmittance of the thin-film samples in the visible region is much smaller than that in the near-infrared region. Specifically, the transmittance is almost $84 \%$ in the transparence region, and it starts to decrease sharply for wavelengths shorter than $1090 \mathrm{~nm}$. The value of transmittance increases after Ta doping; however, the transmittance decreases slightly if the concentration of the Ta dopant continues to increase. The results indicate that Ta doping can improve the transmittance, and the observed decrease of transmittance could be due to the increased absorption of free carriers and the enhanced photon scattering of impurities as the Ta doping level increases [21]. In addition, the absorption edge of the samples shifts toward shorter wavelength (blue-shift) with the increase of Ta doping concentration, implying the increase of band gaps. A related analysis is given in the subsequent calculation section. 

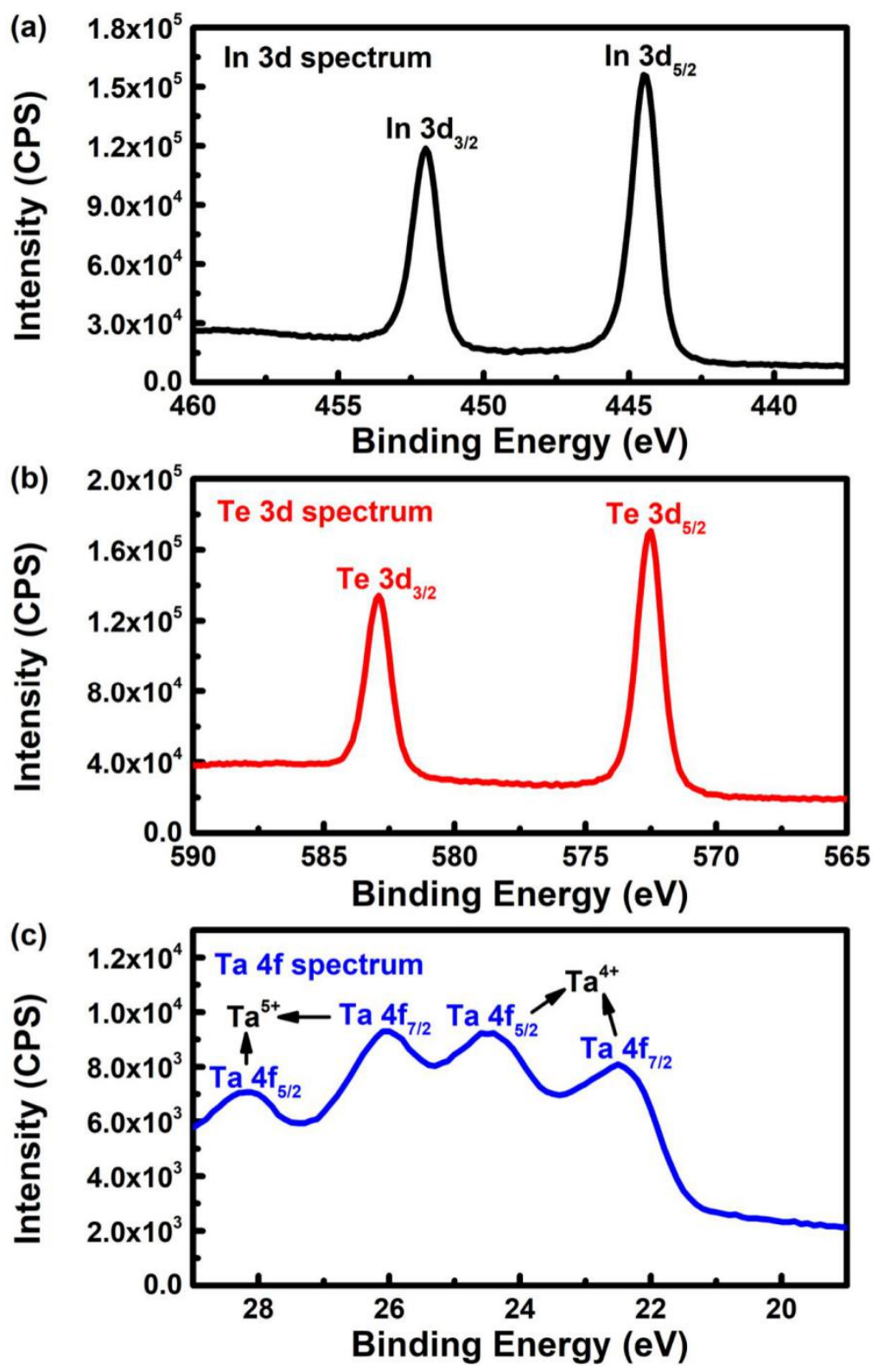

Figure 5. XPS spectra of S3: (a) In 3d, (b) Te 3d, and (c) Ta 4 f regions.
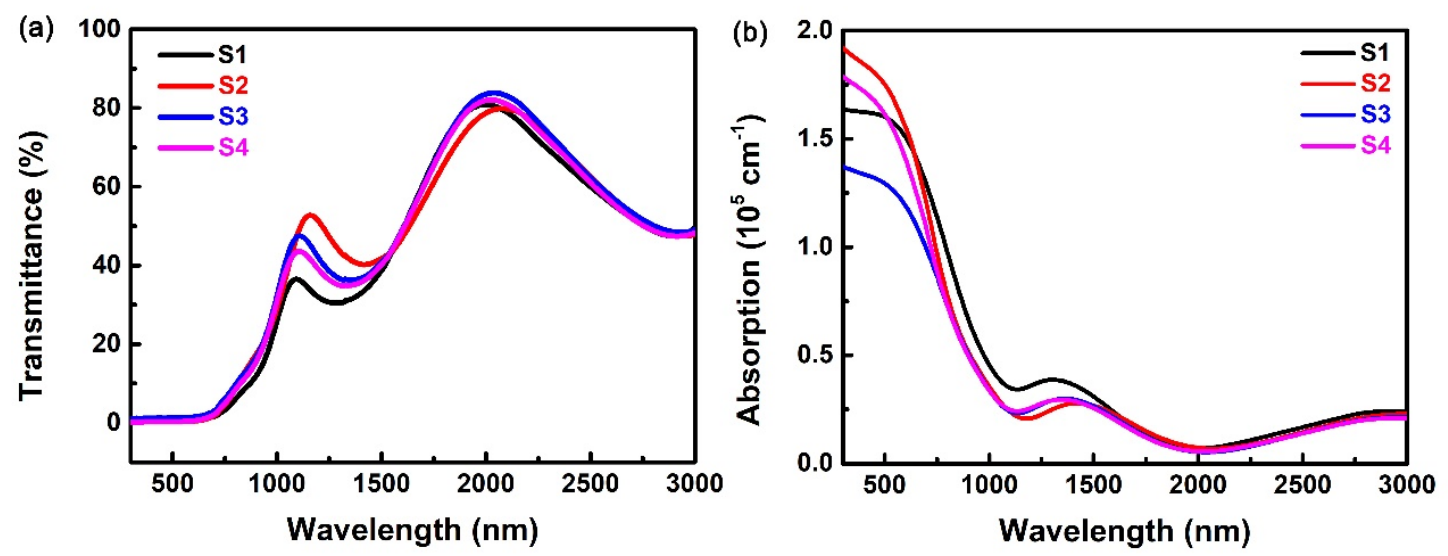

Figure 6. The (a) transmittance and (b) absorption spectra of Ta-doped InTe thin films S1-S4. 
The linear absorption coefficient, $\alpha$, is a key parameter for the characterization of optical band gap and NOA mechanism. It can be calculated from the following Equation [34]:

$$
\alpha=-\ln (T) / d
$$

where $T$ is the transmittance, $d$ is the thickness of the Ta-doped InTe thin film. The calculated linear absorption spectra are plotted in Figure $6 \mathrm{~b}$. In accordance with the transmittance spectra, the values of linear absorption coefficient, $\alpha$, decrease slightly after Ta doping. The absorption region covers the entire visible region and extends up to the near-infrared region, which can be attributed to the presence of different density of localized defect states in the gap.

Based on Tauc's method [35], the optical band gap $\left(E_{g}\right)$ of the Ta-doped InTe film samples can be calculated by using Equation (3):

$$
(\alpha h v)=A\left(h v-E_{g}\right)^{n}
$$

where, $\alpha$ is the calculated linear absorption coefficient from transmittance spectra, $h v$ refers to the incident photon energy, $A$ is a constant which is related to the effective mass and $n$ is a constant that determines the type of optical transitions. For direct transitions in InTe with a narrow gap, $\mathrm{n}$ is equal to $1 / 2$ [36]. The optical band gap $\left(E_{g}\right)$ can be derived from the interception of the linear part of $(\alpha h v)^{2}$ vs. hv for $(\alpha h v)^{2}=0$. As plotted in Figure 7, the sizes of band gap for S1-S4 are 1.71 \pm 0.02 , $1.78 \pm 0.01,1.80 \pm 0.01$ and $1.85 \pm 0.01 \mathrm{eV}$, respectively. It is clear that the optical band gap increases with the increase of the Ta doping. Similar experimental results had also been reported in our previous work, which may be attributed to the Burstein-Moss effect $[15,37]$. That is, the increase of carrier concentration of the doped films and filling of the electronic states in the conduction band can increase the band gap.
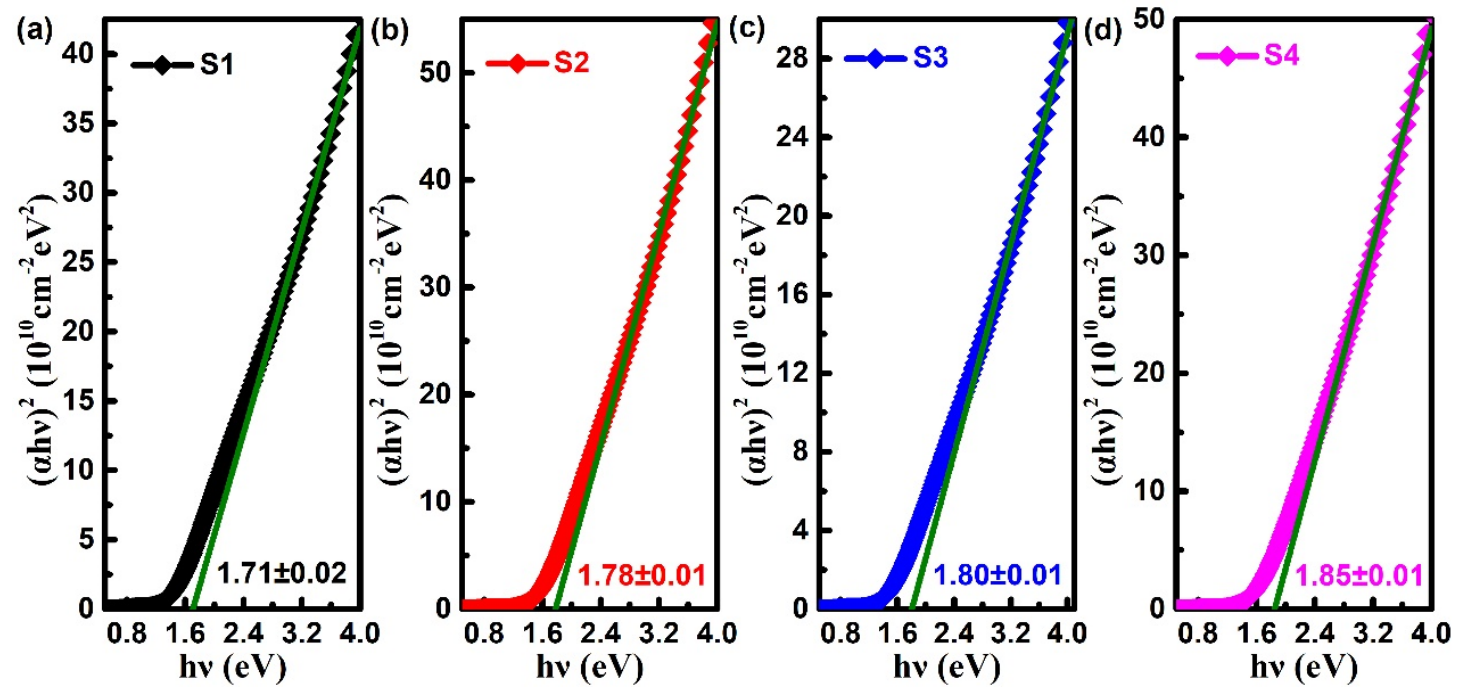

Figure 7. The optical band gaps of Ta-doped InTe thin films: (a) S1, (b) S2, (c) S3, (d) S4.

\subsection{Nonlinear Optical Analysis}

The ultrafast NOA properties of Ta-doped InTe thin films were investigated by a single-beam OA Z-scan method with femtosecond laser pulses at the wavelength of $800 \mathrm{~nm}$ [3]. In the experiment, the samples were sequentially moved along the Z-axis, and the values of intensity-dependent transmittance were recorded. All the samples were measured at a relative low incident power density of $99 \mathrm{GW} / \mathrm{cm}^{2}$, to avoid nonlinear scattering. According to the relationship between transmittance and incident laser intensity, the NOA behaviors in semiconductors can be divided into two types. In a nutshell, saturable absorption (SA) behavior occurs if transmittance increases with increasing incident laser intensity (symmetric peak). The other type shows a symmetric valley, namely, the transmittance 
decreases as the incident laser intensity increases. This optical limiting effect includes two-photon absorption (TPA), multiphoton absorption, and reverse saturable absorption (RSA) [38].

Figure 8 depicts the NOA behaviors of S1-S4, where the dots are experimental data and the curves represent the best fitting results. It is worth noting that the reversible NOA behaviors are caused by the intense laser light, rather than the structural changes of samples or the NOA properties of fused quartz substrates. It can be seen that the normalized transmittance gradually decreases as the sample position approaches the laser focal, whereas the normalized transmittance gradually increases as it moves away from the beam focus, i.e., all the samples exhibit symmetric valleys. This observation is attributed to TPA, since the band gap energy of Ta-doped InTe samples $(1.71-1.85 \mathrm{eV})$ is larger than the energy of one photon $(\sim 1.55 \mathrm{eV})$, but smaller than the energy of two photons $(\sim 3.10 \mathrm{eV})$ [39]. For different samples, the transmittance at the beam focus shows a slight increase at a higher Ta doping concentration. For simplification, the band structure of the Ta-doped InTe thin films is employed to simulate this absorption process, as shown in Figure 9a. An electron in the valence band cannot be directly excited from the valence band to the conduction band by absorbing a photon, because the energy of one single photon is less than the band gap. However, an electron in the valence band can be excited to the conduction band by absorbing two photons.
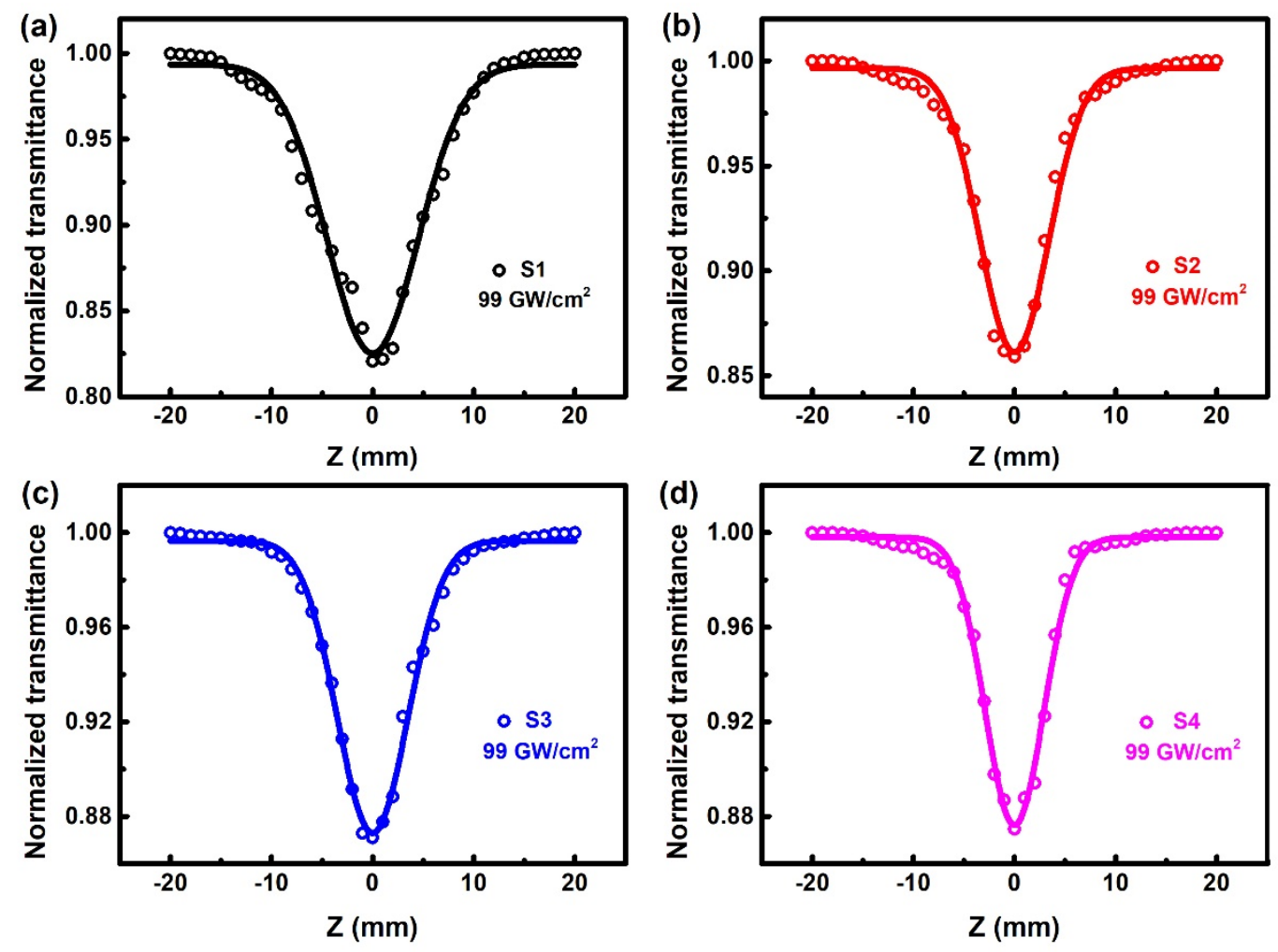

Figure 8. The open-aperture (OA) Z-scan curves of Ta-doped InTe thin films (a) S1, (b) S2, (c) S3, (d) S4 at the excitation of $800 \mathrm{~nm}$ wavelength. 
(a)

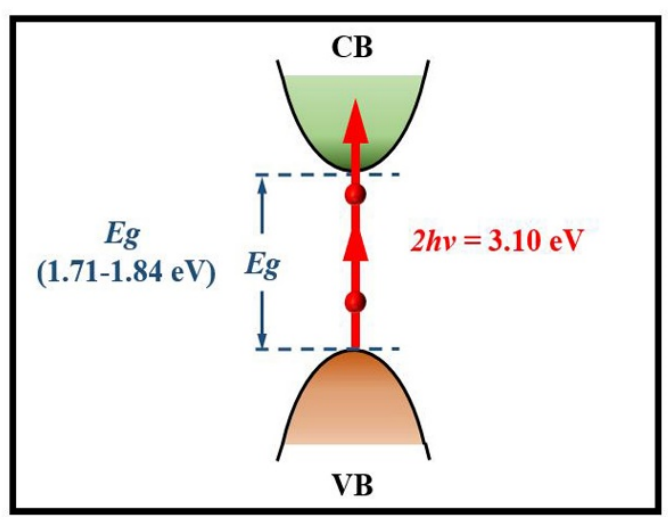

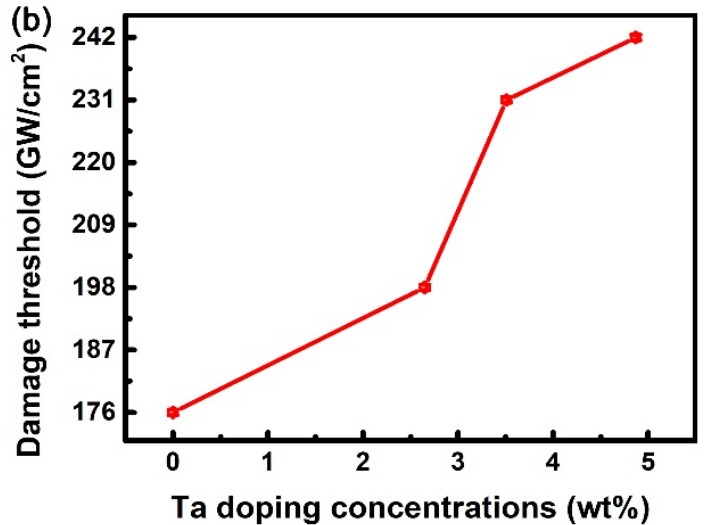

Figure 9. (a) The two-photon absorption (TPA) process and (b) damage threshold of Ta-doped InTe thin films.

The typical OA Z-scan theory by Sheik-Bahae was used to determine the TPA coefficient, $\beta$. It can be deduced by the following equation [23]:

$$
T_{O A}=\sum_{m=0}^{\infty} \frac{\left[-\beta I_{0} L_{e f f} /\left(1+z^{2} / z_{0}^{2}\right)\right]^{m}}{(m+1)^{3 / 2}}
$$

where $T_{O A}$ is the normalized transmittance of the OA Z-scan curve, $I_{0}$ represents the incident laser intensity at the focal point, $z$ indicates the position of the thin films on the axis, $z_{0}$ is the Rayleigh length, and $L_{\text {eff }}$ is the effective thickness of samples, which can be calculated by the following [3]:

$$
L_{e f f}=\frac{1-e^{-\alpha_{0} L}}{\alpha_{0}}
$$

with $\alpha_{0}$ and $L$ as the linear absorption coefficient at the wavelength of $800 \mathrm{~nm}$ and the physical thickness of the sample, respectively. The resultant fitting parameters are listed in Table 4, and the TPA coefficient decreases with the increase of Ta doping concentration. At $800 \mathrm{~nm}$ excitation wavelength, the nonlinear absorption coefficients of $\mathrm{In}_{2} \mathrm{Te}_{3}, \mathrm{Sb}_{2} \mathrm{Se}_{3}, \mathrm{WS}_{2}$, and $\mathrm{MoS}_{2}$ are 805.6, 843.67, -397, and $-136.13 \mathrm{~cm} / \mathrm{GW}$, respectively [3,40-42]. The values of the TPA coefficient are comparable to those of similar chalcogenides. For application purposes, the damage thresholds of Ta-doped InTe films were investigated and illustrated in Figure 9b. The damage thresholds increase from $176 \pm 0.5 \mathrm{GW} / \mathrm{cm}^{2}$ to $242 \pm 0.5 \mathrm{GW} / \mathrm{cm}^{2}$ with increasing Ta doping concentration. This is beneficial to the application in laser-damage-resistance devices. It is worth noting that the doping of Ta increases the damage threshold, while maintaining the high TPA coefficient of InTe material. The TPA behaviors of Ta-doped InTe thin films with large nonlinear absorption coefficient and damage threshold at room temperature are highly advantageous for applications in optical power limiters. Furthermore, the TPA cross-section, $\sigma_{2}$, can be calculated by the following [43]:

$$
\sigma_{2}=\frac{h v \beta}{N_{A} d_{0} \times 10^{-3}}
$$

where $h v$ refers to the incident photon energy, $N_{A}$ is the Avogadro's number, and $d_{0}$ is the molar concentration of the absorbing molecules (in units of M). The TPA cross-section, $\sigma_{2}$, has a unit of $\mathrm{cm}^{4} /$ (photon/s) or $\mathrm{cm}^{4} \mathrm{~s}$. It can also be represented by another informal unit, GM, which is defined by the following:

$$
1 G M=10^{-50} \mathrm{~cm}^{4} \mathrm{~s}
$$


Table 4. Nonlinear optical absorption (NOA) parameters of the Ta-doped InTe thin films measured by OA Z-scan.

\begin{tabular}{cccc}
\hline Sample & $\boldsymbol{L}_{\text {eff }}(\mathbf{n m})$ & $\boldsymbol{\beta}(\mathbf{c m} / \mathrm{GW})$ & $\boldsymbol{\sigma}_{\mathbf{2}}(\mathrm{GM})$ \\
\hline S1 & $97.4 \pm 0.05$ & $526.40 \pm 0.27$ & $89.42 \pm 0.05$ \\
S2 & $117.2 \pm 0.08$ & $343.55 \pm 0.22$ & $58.35 \pm 0.04$ \\
S3 & $125.5 \pm 0.09$ & $293.68 \pm 0.20$ & $49.89 \pm 0.03$ \\
S4 & $127.0 \pm 0.07$ & $281.88 \pm 0.17$ & $47.89 \pm 0.03$ \\
\hline
\end{tabular}

The calculated data are also listed in Table 4. Clearly, the value of $\sigma_{2}$ decreases with increasing Ta doping concentration, which is consistent with the experimental observation. According to He et al., most of the measured values of $\sigma_{2}$ are in the range from $10^{-51}$ to $10^{-46} \mathrm{~cm}^{4} \mathrm{~s}$ [43]. The values of $\sigma_{2}$ are within this reasonable range.

\section{Summary}

In the present work, Ta element was chosen as the dopant to improve the performance of the chalcogenide film. The microstructure, transmittance characteristics, linear optical absorption parameters, and nonlinear optical absorption properties of the InTe films with different Ta doping concentrations were investigated and discussed in detail. These samples were prepared on both fused quartz and Si substrates by a magnetron co-sputtering method, and then they were annealed at $450{ }^{\circ} \mathrm{C}$ for $30 \mathrm{~min}$. X-ray diffraction measurement indicates a crystalline state for all samples, and the crystallinity decreases with increasing Ta doping concentration. Raman spectra analysis also shows good agreement with X-ray diffraction results. The thickness of the samples increases at increasing Ta doping concentration. Chemical analysis reveals that the Ta dopant exists in the InTe films as $\mathrm{Ta}^{5+}$ and $\mathrm{Ta}^{4+}$ states. The transmittance measured via UV-VIS-NIR improves after Ta doping, but it will decrease by further increasing the Ta concentration. Due to the Burstein-Moss effect, the calculated values of optical band gaps increase from $1.71 \pm 0.02 \mathrm{eV}$ to $1.85 \pm 0.01 \mathrm{eV}$ as the Ta doping concentration increases. The NOA responses of the Ta-doped InTe thin films were investigated by using the OA Z-scan technique with the excitation of $800 \mathrm{~nm}$. All samples show two-photon absorption effect. The NOA parameters, such as modulation depth, TPA coefficient, and TPA cross section decrease with increasing Ta doping concentration. In addition, the damage threshold increases at higher Ta dopant concentrations. The performance of InTe films can be adjusted according to Ta doping concentration. Overall, InTe film with low doping concentration could be employed in innovative optoelectronic devices and optical limiters in view of its high transmittance and nonlinear absorption coefficient.

Author Contributions: Writing-original draft preparation, C.L.; writing-review and editing, Y.Y., X.Z., J.S., X.S., H.L., Y.L., H.Z., Y.Z., and J.L.; supervision, J.L. All authors have read and agreed to the published version of the manuscript.

Funding: This work was financially supported by the Natural Science Foundation of Shanghai (Grant Nos. 17ZR1402200, 13ZR1402600), and National Natural Science Foundation of China (Grant Nos. 60578047, 61427815).

Acknowledgments: The authors would like to express their sincere thanks for the financial supports by Natural Science Foundation of Shanghai (Grant Nos. 17ZR1402200, 13ZR1402600), and National Natural Science Foundation of China (Grant Nos. 60578047, 61427815). The authors thank H. B. Zhao, L. Y. Chen and M. Xu for effective backup.

Conflicts of Interest: The authors declare no conflict of interest.

\section{References}

1. Chen, Y.; Sun, L.; Zhou, Y.; Zewdie, G.M.; Deringer, V.L.; Mazzarello, R.; Zhang, W. Chemical understanding of resistance drift suppression in Ge-Sn-Te phase-change memory materials. J. Mater. Chem. C 2020, 8, 71-77. [CrossRef] 
2. Cao, X.; Meng, C.; Li, J.; Wang, J.; Yuan, Y.; Su, J.; Liu, C.; Zhang, X.; Zhang, H.; Wang, J. Characterization of interfacial barrier charging as a resistive switching mechanism in $\mathrm{Ag} / \mathrm{Sb}_{2} \mathrm{Te}_{3} / \mathrm{Ag}$ heterojunctions. Phys. Chem. Chem. Phys. 2018, 20, 18200-18206. [CrossRef]

3. Wang, J.; Jin, F.; Cao, X.; Cheng, S.; Liu, C.; Yuan, Y.; Fang, J.; Zhao, H.; Li, J. $\operatorname{In}_{2} \mathrm{Te}_{3}$ thin films: A promising nonlinear optical material with tunable nonlinear absorption response. RSC Adv. 2016, 6, 103357-103363. [CrossRef]

4. Hajijafarassar, A.; Martinho, F.; Stulen, F.; Grini, S.; Lopez-Marino, S.; Espindola-Rodriguez, M.; Doebeli, M.; Canulescu, S.; Stamate, E.; Gansukh, M.; et al. Monolithic thin-film chalcogenide-silicon tandem solar cells enabled by a diffusion barrier. Sol. Energy Mater. Sol. Cells 2020, 207, 110334. [CrossRef]

5. Huang, W.; Gan, L.; Li, H.; Ma, Y.; Zhai, T. 2D layered group IIIA metal chalcogenides: Synthesis, properties and applications in electronics and optoelectronics. CrystEngComm 2016, 18, 3968-3984. [CrossRef]

6. Hogg, J.H.C.; Sutherland, H.H. Indium Telluride. Acta Crystallogr. Sect. B Struct. Sci. 1976, 32, 2689-2690. [CrossRef]

7. Rajaji, V.; Pal, K.; Sarma, S.C.; Joseph, B.; Peter, S.C.; Waghmare, U.V.; Narayana, C. Pressure induced band inversion, electronic and structural phase transitions in InTe: A combined experimental and theoretical study. Phys. Rev. B 2018, 97, 155158. [CrossRef]

8. Geller, S.; Jayaraman, A.; Hull, G.W. Crystal chemistry and superconductivity of pressure-induced phases in the InTe system. J. Phys. Chem. Solids 1965, 26, 353-361. [CrossRef]

9. Jana, M.K.; Pal, K.; Waghmare, U.V.; Biswas, K. The Origin of ultralow thermal conductivity in InTe: Lone-pair-induced anharmonic rattling. Angew. Chem. Int. Ed. 2016, 55, 7792-7796. [CrossRef]

10. Tritt, T.M. Thermoelectrics run hot and cold. Science 1996, 272, 1276-1277. [CrossRef]

11. Peng, Q.; Xiong, R.; Sa, B.; Zhou, J.; Wen, C.; Wu, B.; Anpo, M.; Sun, Z. Computational mining of photocatalysts for water splitting hydrogen production: Two-dimensional InSe-family monolayers. Catal. Sci. Technol. 2017, 7, 2744-2752. [CrossRef]

12. Peng, Q.; Wang, Z.; Sa, B.; Wu, B.; Sun, Z. Electronic structures and enhanced optical properties of blue phosphorene/transition metal dichalcogenides van der Waals heterostructures. Sci. Rep. 2016, 6, 31994. [CrossRef] [PubMed]

13. Sowjanya, V.; Bangera, K.V.; Shivakumar, G.K. Synthesis of single-phase stoichiometric InTe thin films for opto-electronic applications. Superlattices Microstruct. 2019, 129, 220-225. [CrossRef]

14. Sugiyama, Y.; Chiba, R.; Fujimori, S.; Funakoshi, N. Crystallization process of In-Te alloy-films for optical-recording. J. Non-Cryst. Solids 1990, 122, 83-89. [CrossRef]

15. Yuan, Y.; Liu, C.; Su, J.; Cheng, L.; Fang, J.; Zhang, X.; Sun, Y.; Wu, Y.; Zhang, H.; Li, J. Structural and Optical Properties of Ti-Doped InTe Thin Films. J. Phys. Chem. C 2018, 122, 6267-6272. [CrossRef]

16. Li, H.; Zhou, Z.; Wang, H. Tunable Schottky barrier in InTe/graphene van der Waals heterostructure. Nanotechnology 2020, 31, 335201. [CrossRef]

17. Back, S.Y.; Kim, Y.-K.; Cho, H.; Han, M.-K.; Kim, S.J.; Rhyee, J.S. Temperature-induced lifshitz transition and charge density wave in InTe ${ }_{1-\delta}$ thermoelectric materials. ACS Appl. Energy Mater. 2020, 3, 3628-3636. [CrossRef]

18. Pan, S.; Liu, H.; Li, Z.; You, L.; Dai, S.; Yang, J.; Guo, K.; Luo, J. Enhancement of the thermoelectric performance of InTe via introducing Cd dopant and regulating the annealing time. J. Alloys Compd. 2020, 813, 152210. [CrossRef]

19. Cheng, S.; Wei, S.; Yi, X.; Wang, J.; Liu, C.; Li, J.; Yang, T. Investigations on phase change characteristics of Ti-doped $\mathrm{Ge}_{2} \mathrm{Sb}_{2} \mathrm{Te}_{5}$ system. J. Phys. D Appl. Phys. 2015, 48, 475108. [CrossRef]

20. Suh, J.; Sarkar, T.; Choe, H.S.; Park, J.; Venkatesan, T.; Wu, J. Compensated thermal conductivity of metallically conductive Ta-doped $\mathrm{TiO}_{2}$. Appl. Phys. Lett. 2018, 113, 022103. [CrossRef]

21. Zhao, W.; He, L.; Feng, X.; Luan, C.; Ma, J. Structural, electrical and optical properties of epitaxial Ta-doped titania films by MOCVD. CrystEngComm 2018, 20, 5395-5401. [CrossRef]

22. Music, D.; Chen, X.; Holzapfel, D.M.; Bilyalova, H.M.; Helvaci, M.; Heymann, A.O.D.; Aghda, S.K.; Maron, T.; Ravensburg, A.L.; Saelker, J.A.; et al. Physical origin of inertness of Ta contacts on $\mathrm{Bi}_{2} \mathrm{Te}_{3}$. J. Appl. Phys. 2018, 124, 185106. [CrossRef]

23. Sheikbahae, M.; Said, A.A.; Wei, T.H.; Hagan, D.J.; Vanstryland, E.W. Sensitive measurement of optical nonlinearities using a single beam. IEEE J. Quantum Electron. 1990, 26, 760-769. [CrossRef]

24. Kavitha, V.S.; Suresh, S.; Chalana, S.R.; Pillai, V.P.M. Luminescent Ta doped $\mathrm{WO}_{3}$ thin films as a probable candidate for excitonic solar cell applications. Appl. Surf. Sci. 2019, 466, 289-300. [CrossRef]

25. Cullity, B.D. Elements of X-Ray Diffraction; Addison-Wesley Publishing Co., Inc.: Boston, MA, USA, 1956.

26. Pine, A.S.; Dresselhaus, G. Raman Spectra and Lattice Dynamics of Tellurium. Phys. Rev. B 1971, 4, 356-371. [CrossRef] 
27. Sun, J.; Nie, Q.; Wang, X.; Dai, S.; Zhang, X.; Bureau, B.; Boussard, C.; Conseil, C.; Ma, H. Structural investigation of Te-based chalcogenide glasses using Raman spectroscopy. Infrared Phys. Technol. 2012, 55, 316-319. [CrossRef]

28. Nizametdinova, M.A. Raman Spectrum of InTe and TlSe Single Crystals. Phys. Status Solidi B 1980, 97, K9-K12. [CrossRef]

29. Golding, T.D.; Boyd, P.R.; Martinka, M.; Amirtharaj, P.M.; Dinan, J.H. Molecular-beam-epitaxial growth and characterization of $\mathrm{In}_{2} \mathrm{Te}_{3}$. J. Appl. Phys. 1989, 65, 1936-1941. [CrossRef]

30. Lakhe, M.; Chaure, N.B. Characterization of electrochemically deposited CuInTe 2 thin films for solar cell applications. Sol. Energy Mater. Sol. Cells 2014, 123, 122-129. [CrossRef]

31. Tkalich, A.K.; Demin, V.N.; Zlomanov, V.P. Oxidation States of In in Pb ${ }_{1-x} \operatorname{In}_{x}$ Te. J. Solid State Chem. 1995, 116, 33-36. [CrossRef]

32. Glendening, E.D.; Landis, C.R.; Weinhold, F. Resonance theory reboot. J. Am. Chem. Soc. 2019, 141, $4156-4166$. [CrossRef] [PubMed]

33. Ohno, Y. The scanning-tunneling microscopy, the X-ray photoelectron spectroscopy, the inner-shell-electron energy-loss spectroscopy studies of $\mathrm{MTe}_{2}$ and $\mathrm{M}_{3} \mathrm{SiTe}_{6}(\mathrm{M}=\mathrm{Nb}$ and $\mathrm{Ta})$. J. Solid State Chem. 1999, 142, 63-73. [CrossRef]

34. Chen, C.; Li, W.; Zhou, Y.; Chen, C.; Luo, M.; Liu, X.; Zeng, K.; Yang, B.; Zhang, C.; Han, J.; et al. Optical properties of amorphous and polycrystalline $\mathrm{Sb}_{2} \mathrm{Se}_{3}$ thin films prepared by thermal evaporation. Appl. Phys. Lett. 2015, 107, 043905. [CrossRef]

35. Tauc, J.; Grigorovici, R.; Vancu, A. Optical properties and electronic structure of amorphous germanium. Phys. Status Solidi 1966, 15, 627-637. [CrossRef]

36. Pal, S.; Bose, D.N. Growth, characterisation and electrical anisotropy in layered chalcogenides GaTe and InTe. Solid State Commun. 1996, 97, 725-729. [CrossRef]

37. Shelke, V.; Bhole, M.P.; Patil, D.S. Effect of open air annealing on spin coated aluminum doped ZnO nanostructure. Mater. Chem. Phys. 2013, 141, 81-88. [CrossRef]

38. Karatay, A.; Yaglioglu, H.G.; Elmali, A.; Parlak, M.; Karaagac, H. Thickness-dependent nonlinear absorption behaviors in polycrystalline ZnSe thin films. Opt. Commun. 2012, 285, 1471-1475. [CrossRef]

39. Vanstryland, E.W.; Woodall, M.A.; Vanherzeele, H.; Soileau, M.J. Energy band-gap dependence of two-photon absorption. Opt. Lett. 1985, 10, 490-492. [CrossRef]

40. Liu, C.; Yuan, Y.; Cheng, L.; Su, J.; Zhang, X.; Li, X.; Zhang, H.; Zhang, X.; Li, J. Tunable nonlinear optical absorption in amorphous and crystalline $\mathrm{Sb}_{2} \mathrm{Se}_{3}$ thin films. J. Alloys Compd. 2019, 791, 753-760. [CrossRef]

41. Zhang, S.; Dong, N.; McEvoy, N.; O’Brien, M.; Winters, S.; Berner, N.C.; Yim, C.; Li, Y.; Zhang, X.; Chen, Z.; et al. Direct observation of degenerate two-photon absorption and its saturation in $\mathrm{WS}_{2}$ and $\mathrm{MoS}_{2}$ nono layer and few-layer films. ACS Nano 2015, 9, 7142-7150. [CrossRef]

42. He, M.; Quan, C.; He, C.; Huang, Y.; Zhu, L.; Yao, Z.; Zhang, S.; Bai, J.; Xu, X. Enhanced nonlinear saturable absorption of $\mathrm{MoS}_{2}$ /graphene nanocomposite films. J. Phys. Chem. C 2017, 121, 27147-27153. [CrossRef]

43. He, G.S.; Tan, L.-S.; Zheng, Q.; Prasad, P.N. Multiphoton absorbing materials: Molecular designs, characterizations, and applications. Chem. Rev. 2008, 108, 1245-1330. [CrossRef] [PubMed]

(C) 2020 by the authors. Licensee MDPI, Basel, Switzerland. This article is an open access article distributed under the terms and conditions of the Creative Commons Attribution (CC BY) license (http://creativecommons.org/licenses/by/4.0/). 